\title{
Sofosbuvir for the treatment of chronic hepatitis C: between current evidence and future perspectives
}

This article was published in the following Dove Press journal:

Hepatic Medicine: Evidence and Research

29 April 2014

Number of times this article has been viewed

\author{
Elisabetta Degasperi \\ Alessio Aghemo \\ Division of Gastroenterology and \\ Hepatology, AM and M Migliavacca \\ Center, Fondazione IRCCS Ca' \\ Granda Maggiore Hospital, University \\ of Milan, Milan, Italy
}

\begin{abstract}
In recent years, clinical research in the field of new treatments for chronic hepatitis $\mathrm{C}$ (HCV) has been devoted to developing regimens based on direct-acting antivirals (DAAs), with the goal of increasing treatment efficacy and improving tolerability and safety. This can be achieved by Peginterferon (PegIFN)-free anti-HCV regimens, as PegIFN is responsible for many side effects and limits treatment access due to contraindications in some patient categories. Sofosbuvir (SOF) is the first compound to enter the market with IFN-free combination regimens; it belongs to the nucleotide inhibitors of viral polymerase NS5B and acts as a chain terminator during the HCV replication process, exhibiting pan-genotypic antiviral activity with a high barrier to resistance. Clinical trials in $\mathrm{HCV}$ genotype $2 / 3$ patients have demonstrated optimal efficacy in $\mathrm{HCV}-2$, where the combination SOF/ribavirin (Rbv) for 12 weeks resulted in $>90 \%$ sustained virological response (SVR) rates, while HCV-3 patients with advanced liver fibrosis and previous failure to PegIFN plus Rbv therapy still require individualized and optimized treatment strategies. Historically difficult-to-treat genotypes HCV-1, -4-6 can benefit from reduced duration of PegIFN plus SOF and Rbv, while IFN-free regimens in these patients will be based on SOF in combination with other DAA classes. Due to an optimal tolerability and safety profile with no significant drug-to-drug interactions, SOF is currently undergoing clinical trials in the setting of pre- and post-liver transplantation and HIV-coinfected patients, with the objective to address the until now unmet need for safe and efficient treatment in these populations. This article provides an overview of SOF features and the main clinical trials, discussing key results and potential future developments.
\end{abstract}

Keywords: sofosbuvir, hepatitis C, antiviral treatment

\section{Introduction}

Chronic infection with hepatitis $\mathrm{C}$ virus (HCV) affects more than 170 million people worldwide and is a leading cause of anticipated liver-related death due to the development of cirrhosis and its complications. ${ }^{1}$ In the last 10 years, standard of care anti-HCV treatment has been founded on the combination of Peginterferon (Peg-IFN) plus ribavirin ( $\mathrm{Rbv}$ ), whose main disadvantages were suboptimal rates of sustained virological response (SVR) in difficult-to-treat patients (HCV genotype 1-4, advanced liver fibrosis) and, most of all, side effects profile resulting in poor tolerability and treatment contraindication in some patient subsets (decompensated liver disease and autoimmune disorders). ${ }^{2}$ The recent availability of culture cell models provided deeper insight in understanding HCV life cycle and was the basis for the development of new drugs targeting non-structural HCV proteins involved in viral replication process, such as NS3 and NS5A/B (Figure 1 and Table 1). Direct-acting antivirals
Correspondence: Alessio Aghemo Division of Gastroenterology and Hepatology, Fondazione IRCCS Ca' Granda Ospedale Maggiore Policlinico, Università degli Studi di Milano,

Via F Sforza 35, Milan, Italy

Tel +390255035432

$\mathrm{Fax}+390250320410$

Email alessio.aghemo@policlinico.mi.it 


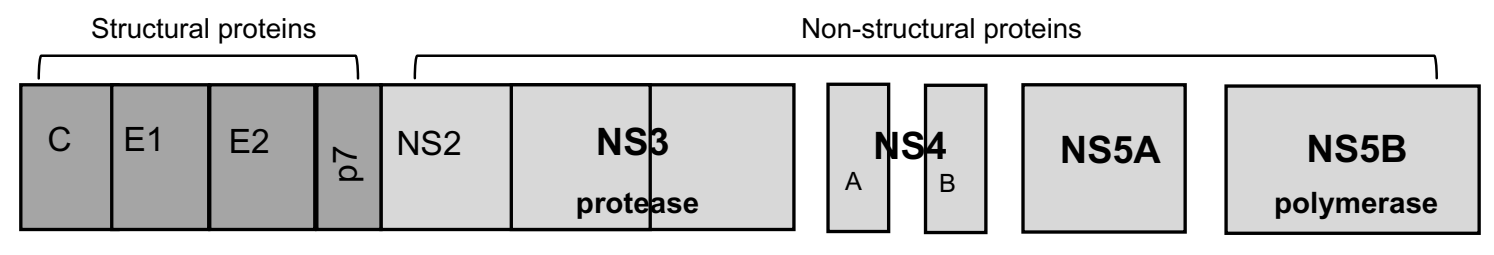

Figure I HCV genomic structure. After hepatocyte binding and cell internalization, HCV-RNA is released and translated into a polyprotein containing structural and nonstructural HCV proteins. NS3 serine protease and a cofactor NS4A allow for post-translational cleavage and proteolysis of the polyprotein to release NS5A and NS5B that start the viral replication process. Inhibition of NS3, NS5A/B by different DAA classes results in impaired HCV replication.

Abbreviations: DAA, direct-acting antivirals; HCV, hepatitis C virus; RNA, ribonucleic acid.

(DAAs) promised to open a new era in treating chronic $\mathrm{HCV}$ infection by increasing SVR rates, providing shortened and simplified regimens while also minimizing treatment-related side effects. First-generation NS3 protease inhibitors telaprevir (TVR) and boceprevir (BOC), approved since 2011 as the new standard of care treatment for HCV genotype 1 patients, have only partially met these expectations: indeed, in Phase III trials and especially in larger real-life cohorts, efficacy of TVR/BOC has been shown to be largely dependent on Peginterferon (PegIFN) plus ribavirin (Rbv) backbone antiviral activity, with disappointing SVR rates in difficultto-treat patients such as previous non-responders to dual therapy. ${ }^{3}$ Moreover, an unfavorable safety profile with high rates of side effects, especially in patients with advanced liver fibrosis, was the main concern with NS3 protease inhibitors, leading to an intensified monitoring schedule and necessity for careful patient selection in order to prevent serious adverse events. ${ }^{4}$

In the meantime, the development of many new compounds belonging to different antiviral classes is expected to overcome the first-generation DAAs by providing a combination of all-oral, IFN-free regimens, that will also allow extended treatment in patients formerly contraindicated or

Table I Main DAA targets and drug classes

\begin{tabular}{ll}
\hline Target & Drug name \\
\hline NS3 (protease) & Telaprevir \\
Ist wave & Boceprevir \\
2nd wave & Simeprevir \\
& Faldaprevir \\
& Asunaprevir \\
& ABT-450 \\
NS5A & Vaniprevir \\
& Daclatasvir \\
& Ledipasvir \\
NS5B (polymerase) & ABT-267 \\
Nucleotidic & \\
Non-nucleotidic & Sofosbuvir \\
\hline
\end{tabular}

Abbreviation: DAA, direct-acting antivirals. intolerant to IFN-based therapies. ${ }^{5}$ This review will focus on sofosbuvir, formerly named GS-7977, an NS5B polymerase nucleotide inhibitor, whose US Food and Drug Administration (FDA) and European Medicines Agency (EMA) approvals have been granted at the end of 2013 and at the beginning of 2014, respectively.

\section{Pharmacokinetic features}

Sofosbuvir (SOF) is an HCV-specific nucleotide inhibitor of viral NS5B polymerase that acts as an chain terminator when incorporated as a substrate by RNA polymerase in the nascent HCV-RNA genome, leading to inhibition of viral replication. Due to the high conservation of the enzyme active site targeted by this drug class, SOF displays pangenotypic antiviral activity against all $\mathrm{HCV}$ genotypes and also has a high barrier to resistance. ${ }^{6}$ Cell culture replicon data demonstrated $\mathrm{EC}_{50}$ values slightly higher for HCV$1 \mathrm{~b}(110 \mathrm{nM})$ and HCV-3 $(50 \mathrm{nM})$ compared to HCV-1a $(40 \mathrm{nM})$ and HCV-2 $(15 \mathrm{nM})$ replicons. SOF is administered once daily, via oral tablets $(400 \mathrm{mg}$ ), with no dependence on food intake. It enters the hepatocytes as a prodrug of uridine monophosphate and is phosphorylated within the cells to active triphosphate form. Dephosphorylation of the active molecule results in the formation of the metabolite GS-331007, which lacks anti-HCV activity; GS-331007 is the main circulating metabolite of SOF and undergoes renal elimination. The median half-lives of SOF and GS-331007 are 0.4 and 27 hours, respectively. Population pharmacokinetic (PK) models developed for GS-331007 and SOF revealed that demographic variables such as age, sex, BMI, race, common concomitant medications, and cirrhosis did not influence GS-331007 or SOF exposure. ${ }^{?}$ PK studies in patients with renal impairment demonstrated that dose modification of SOF is not required if creatinine clearance is $\geq 30 \mathrm{~mL} /$ minute; however, SOF safety has not been established in patients with severe renal impairment or end-stage renal disease, so dose recommendations for these populations cannot be provided. Concerning patients with hepatic impairment, PK analyses showed that cirrhosis 
had no clinically relevant effect on SOF and GS-331007 exposure, so no dose adjustment is recommended in patients with mild-to-severe hepatic impairment.

\section{Sofosbuvir in HCV-2 and -3 patients: IFN-free regimens}

The main goals in SOF development for historically "easyto-treat" genotypes were to shorten treatment duration, but most of all, to improve tolerability through an IFN-free regimen. The ELECTRON trial was the first Phase II study in treatment-naïve patients to explore an IFN-free arm of SOF/Rbv for 12 weeks compared to three arms of variable PegIFN duration (4/8/12 weeks), with $100 \%$ SVR in both the PegIFN-based and IFN-free arms. ${ }^{8}$ This was the first proof of concept that an all-oral, IFN-free regimen was feasible for HCV-2 and -3 patients; conversely, the ELECTRON trial also demonstrated that the key role of Rbv could not be superseded, as a SOF monotherapy 12-week arm reported SVR of $60 \%$ due to a $40 \%$ virological relapse rate. Based on these results, the $\mathrm{SOF} / \mathrm{Rbv}$ combination administered for 12 weeks was chosen to be further evaluated in Phase III trials. The FISSION trial was an open-label, non-inferiority study enrolling $499 \mathrm{HCV}-2 / 3$ naïve patients receiving SOF/Rbv for 12 weeks in comparison with a standard of care arm (PegIFN/ Rbv for 24 weeks). HCV-3 patients accounted for the majority of the study population ( $72 \%$ ), and $20 \%$ cirrhotic patients were included in the study. The trial met the non-inferiority endpoint, showing an overall SVR rate of $67 \%$, although with large differences between the HCV-2 and -3 patients: indeed, when splitting SVR rates according to genotype, HCV-2 patients achieved 93\% SVR, compared to only $56 \%$ in $\mathrm{HCV}-3$ patients. At the multivariate analysis, liver fibrosis was identified as the main predictor of treatment failure only in HCV-3, where the presence of cirrhosis translated to SVR falling to $34 \%$, while fibrosis did not influence SVR rates in HCV-2 patients (91\%). ${ }^{9}$ Following the FISSION trial, it was clear that HCV-3 patients with advanced liver fibrosis could now be identified as the new "difficult to treat" patient group even with more potent regimens.

Tolerability was the main aim of the POSITRON trial, which evaluated the safety and efficacy of SOF/Rbv for 12 weeks in $278 \mathrm{HCV}-2 / 3$ patients that were previously intolerant, unwilling, or contraindicated to PegIFN/Rbv. The study design was placebo-controlled, and similarly to the FISSION trial, allowed enrollment of cirrhotic patients (18\%). While the study confirmed overall high efficacy with $78 \%$ SVR, again, cure rates were extremely different according to HCV genotype ( $97 \%$ in $\mathrm{HCV}-2$ versus [vs] $61 \%$ in $\mathrm{HCV}-3$ ) and fell to only $21 \%$ in HCV-3 cirrhotic patients. Concerning safety, the combination of SOF/Rbv showed an optimal tolerability profile: the most frequent adverse events were fatigue (44\%), nausea (22\%), headache (21\%), insomnia (19\%), and pruritus (11\%), mainly consistent with Rbv. Hemoglobin decline $<10 \mathrm{~g} / \mathrm{dL}$ occurred in only $7 \%$ of patients $(<8.5 \mathrm{~g} / \mathrm{dL}$ in $1 \%$ ), while no reduction in platelets and neutrophil values were reported. The discontinuation rate due to adverse events was only $2 \% .^{10}$

The efficacy of the SOF/Rbv IFN-free combination in patients with a previous treatment failure to PegIFN/ Rbv was investigated in the FUSION trial, which enrolled $201 \mathrm{HCV}-2$ and -3 patients (76\% with a prior relapse) receiving $\mathrm{SOF} / \mathrm{Rbv}$ for 12 or 16 weeks. Thirty-five percent of patients included in the study had compensated cirrhosis and the majority enrolled were $\mathrm{HCV}-3$ patients $(62 \%)$. Overall SVR in treatment experienced patients with SOF/ Rbv was significantly lower in the 12-week compared to 16-week arms ( $50 \%$ vs $73 \%$ ), with huge differences between HCV- 2 and HCV- 3 patients ( $86 \%$ vs $94 \%$ in $\mathrm{HCV}-2,30 \%$ vs $62 \%$ in HCV-3). Cirrhosis and HCV genotype 3 were confirmed as predictors of treatment failure: indeed, in the two treatment arms (12/16 weeks) SVR rates were respectively $96 \% / 100 \%$ in non-cirrhotic vs $60 \% / 78 \%$ in cirrhotic HCV-2 patients, while in HCV-3 patients, SVR decreased to $37 \% / 63 \%$ in non-cirrhotics vs $19 \% / 61 \%$ in cirrhotics. ${ }^{10}$ Consequently, the FUSION trial demonstrated that for $\mathrm{HCV}-2$ the combination of SOF/Rbv provided an all-oral regimen with high efficacy and the possibility of shortening treatment to 12 weeks even in previously treatment-failure patients, while for HCV-3 treatment, extension to 16 weeks translated to higher SVR rates. However, the results were still largely disappointing in patients with advanced liver fibrosis. Results from the SOF trials in $\mathrm{HCV}-2 / 3$ patients are summarized in Figure 2.

Some ongoing studies are evaluating new strategies to increase efficacy in HCV-3 patients: 1) the possibility of extending treatment duration to 24 weeks with the SOF/Rbv regimen; 2) the addition of PegIFN; and 3) the combination of more DAAs. According to the first strategy, the VALENCE trial evaluated efficacy of a 24-week course of $\mathrm{SOF} / \mathrm{Rbv}$ in naïve or treatment-experienced HCV-3 patients. The study results, recently presented to the AASLD Meeting, reported overall 85\% SVR12 rates; however, when stratifying patients according to liver staging and previous treatment course, SVR rates were $92 \%-94 \%$ in naïve and $87 \%$ in treatment-experienced non-cirrhotics, while cirrhotic patients with a previous treatment failure 
HCV-2
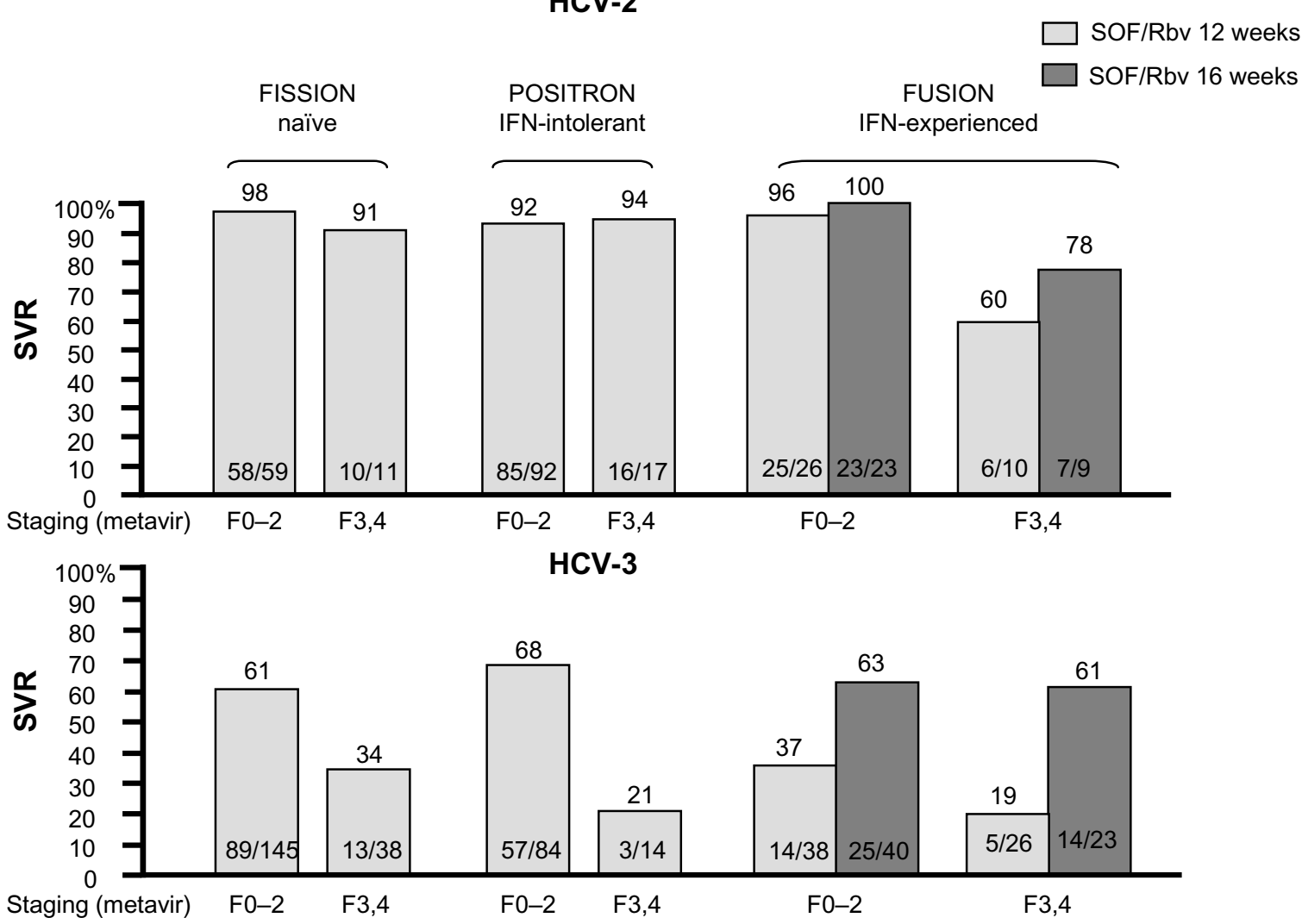

Figure 2 Sofosbuvir trials in HCV-2 and -3 patients.

Abbreviations: HCV, hepatitis C virus; IFN, interferon; Rbv, ribavirin; SOF, sofosbuvir; SVR, sustained virological response.

achieved only $60 \%$ SVR. ${ }^{11}$ These data showed that these patients still remain difficult-to-cure even with an extended treatment, and consequently, could require the addition of an immunomodulatory drug like PegIFN or another DAA. Indeed, the LONESTAR-2 trial, which investigated the combination of SOF/PegIFN/Rbv for 12 weeks, reported 83\% SVR rates in HCV-3 cirrhotic patients previously treatment experienced, although limited patient numbers need to be confirmed in larger cohorts. ${ }^{12}$ Concerning the combination of more DAAs targeting different viral proteins, the ELECTRON trial evaluated the combination of SOF/ ledipasvir (LDV, inhibitor of NS5A)/Rbv for 12 weeks in a Phase II study in naïve non-cirrhotic patients, reporting overall $80 \%$ SVR12 rates. $^{13}$

\section{Sofosbuvir in HCV-I-6 patients: IFN-sparing regimens}

Phase II studies in HCV-1-6 genotypes tested the possibility of developing an IFN-free combination for "difficult to treat" genotypes, or at least, to spare PegIFN administration in terms of limited treatment duration. The Phase IIb open-label ATOMIC trial enrolled 316 naïve non-cirrhotic HCV genotype 1 and 16 genotype 4-6 patients receiving SOF in combination with PegIFN/Rbv for 12 or 24 weeks; a third arm received SOF/PegIFN/Rbv for 12 weeks and then $\mathrm{SOF} \pm \mathrm{Rbv}$ for an additional 12 weeks. SVR12 rates in the three arms did not significantly differ, being respectively $90 \%, 92 \%$, and $91 \%$, and no viral breakthrough was observed, so that the 12-week duration of SOF/PegIFN/Rbv demonstrated the best efficacy/cost ratio in treating naïve non-cirrhotic patients. ${ }^{14}$ An IFN-free regimen for HCV-1 patients was tested in the ELECTRON trial, originally enrolling only HCV-2 and -3 patients, where a protocol amendment led to the enrollment of $35 \mathrm{HCV}-1$ patients ( $90 \%$ subtype 1a), ten previously nullresponders and 25 naïve to PegIFN/Rbv therapy, receiving an IFN-free regimen of SOF/Rbv for 12 weeks. The two patient groups showed dramatically different SVR rates, as only $10 \%$ of null responders achieved SVR, compared to $84 \%$ of naïve patients, with relapse rates being respectively $84 \%$ and $26 \% .{ }^{8}$ This was the demonstration that to achieve high SVR rates in HCV-1 patients, SOF needs to be combined with PegIFN and Rbv.

NEUTRINO was the first SOF Phase III trial in $327 \mathrm{HCV}$ genotype 1,4-6 naïve patients with the aim to evaluate the safety and efficacy of the combination regimen SOF/PegIFN/ Rbv for 12 weeks. Patient baseline demography included 
89\% HCV-1 (69\% subtype 1a), 9\% HCV-4, and 2\% HCV-5,6 patients; IL28B non-CC genotype accounted for the majority (71\%) of patients. NEUTRINO allowed enrollment of cirrhotic patients, who represented $17 \%$ of study population.

The combination of SOF/PegIFN/Rbv for 12 weeks demonstrated high rates of on-treatment response, with $91 \%$ of patients achieving HCV-RNA undetectability at week 2 and $99 \%$ at week 4 , which resulted in an overall SVR rate of $90 \%$. When stratifying patients according to HCV genotype, SVR rates were $89 \%$ in $\mathrm{HCV}-1,96 \%$ in $\mathrm{HCV}-4$, and $100 \%$ in $\mathrm{HCV}$ 5,6 (despite the small number of HCV-4-6 patients included). HCV subtype 1a achieved higher SVR rates than $1 \mathrm{~b}(92 \%$ vs $82 \%$ ). At multivariate analysis, the IL28B genotype non-CC and cirrhosis were found to be predictors of treatment failure: indeed SVR rates were $87 \%$ in non-CC vs $98 \%$ in CC patients ( $P=0.006$, OR: 7.989 ), while cirrhotic patients achieved overall $80 \%$ SVR vs $92 \%$ in non-cirrhotics ( $P=0.002$, OR: 3.924$)$. Viral kinetics on-treatment did not significantly differ in cirrhotics vs non-cirrhotic patients, all patients achieved high on-treatment response rates; relapse accounted for all treatment failures, as no viral breakthrough was observed. Concerning safety profile, the most common side effects reported were fatigue (59\%), headache (36\%), nausea (34\%), and insomnia (25\%), which are mainly consistent with Rbv or PegIFN safety profiles. Anemia with $\mathrm{Hb}<10 \mathrm{~g} / \mathrm{dL}$ was observed in $23 \%$, while only $2 \%$ of patients had $\mathrm{Hb}<8.5 \mathrm{~g} / \mathrm{dL}$. Discontinuation rates due to adverse events were lower than $2 \%{ }^{9}$

Results from the SOF Phase III trials are summarized in Table 2.

\section{SOF in combination with other DAAs: IFN-free regimens for HCV I-4}

The possibility of developing an IFN-free regimen for difficult-to-treat genotypes requires the combination of different DAA classes to provide high antiviral efficacy as well as a high barrier to resistance. According to these features, the ideal regimen could combine nucleotide inhibitors of NS5B such as SOF, compounds with the highest resistance barrier, plus NS5A or NS3 inhibitors that are characterized by the strongest viral suppression. The first combination of SOF plus NS5A inhibitors was evaluated in a Phase II study in treatment-naïve non-cirrhotic $\mathrm{HCV}-1$ patients who received SOF in combination with daclatasvir (DCV) $60 \mathrm{mg}$ QD \pm Rbv for 12 or 24 weeks, reporting $100 \%$ SVR in all treatment arms. In the same study, a cohort of 41 patients with a previous treatment failure to protease inhibitors BOC or TVR were treated with $\mathrm{SOF} / \mathrm{DCV} \pm \mathrm{Rbv}$ for 24 weeks, showing $100 \%$ SVR rates as well. ${ }^{15}$ This study confirmed that the possible emergence of resistance-associated variants (RAVs) to protease inhibitors lacks cross-resistance with other DAA classes and could be efficiently cured by more potent drug combinations. Another promising IFN-free regimen is SOF in combination with the NS5A inhibitor LDV $90 \mathrm{mg}$ QD \pm Rbv, which has been evaluated by the LONESTAR trial in HCV-1 naïve and TVR/BOC-experienced patients: the study demonstrated that 8 weeks of SOF/LDV were sufficient to achieve respectively $100 \%$ and $95 \%$ SVR rates with or without Rbv. In the TVR/BOC-experienced population, where $55 \%$ of patients had cirrhosis, SVR rates were respectively $100 \%$ and $95 \%$ in arms receiving a 12-week treatment course with or without Rbv (Figure 3). Resistance analysis showed S282T NS5B mutation and multiple NS5A RAVs detected at relapse in one patient receiving $\mathrm{SOF} / \mathrm{LDV}$ for 8 weeks; the patient subsequently achieved SVR12 after retreatment with SOF/LDV + Rbv. R155K was the most common RAV in PI-experienced patients; however, all patients with RAVs achieved SVR. ${ }^{16}$ The ELECTRON Phase II study is currently evaluating the efficacy of $\mathrm{SOF} / \mathrm{LDV} \pm \mathrm{Rbv}$ in additional cohorts, including $\mathrm{HCV}-1$ cirrhotics that were prior

Table 2 Summary of sofosbuvir Phase III trials

\begin{tabular}{|c|c|c|c|c|c|}
\hline Trial & $\begin{array}{l}\text { HCV } \\
\text { genotype }\end{array}$ & $\begin{array}{l}\text { Patient population } \\
\text { (\% cirrhosis) }\end{array}$ & $\begin{array}{l}\text { Treatment } \\
\text { status }\end{array}$ & Regimen & Key messages \\
\hline FISSION & $2-3$ & $499(20 \%)$ & Naïve & $\mathrm{SOF}+\mathrm{Rbv} 12 \mathrm{~W}$ & $\begin{array}{l}\text { - SOF + Rbv high overall efficacy } \\
\text { - Lower SVR rates in HCV-3 cirrhotics }\end{array}$ \\
\hline POSITRON & $2-3$ & $278(15 \%)$ & $\begin{array}{l}\text { IFN intolerant, } \\
\text { ineligible/unwilling }\end{array}$ & $\mathrm{SOF}+\mathrm{Rbv} 12 \mathrm{~W}$ & - Optimal tolerability, low side effects \\
\hline FUSION & $2-3$ & $201(34 \%)$ & IFN experienced & $\mathrm{SOF}+\mathrm{Rbv} 12 / 16 \mathrm{~W}$ & $\begin{array}{l}\text { - Suboptimal SVR in HCV-3 cirrhotics } \\
\text { - Better SVR rates in HCV-3 with } \\
\text { extended } 16 \text {-week course }\end{array}$ \\
\hline NEUTRINO & $1,4-6$ & 327 (17\%) & Naïve & $\begin{array}{l}\mathrm{SOF}+\mathrm{PegIFN}+\mathrm{Rbv} \\
\mathrm{I} 2 \mathrm{~W}\end{array}$ & $\begin{array}{l}\text { - High efficacy (>90\%) across all } \\
\text { genotypes }\end{array}$ \\
\hline
\end{tabular}

Abbreviations: HCV, hepatitis C virus; IFN, interferon; PegIFN, Peginterferon; Rbv, ribavirin; SOF, sofosbuvir; SVR, sustained virological response; W, weeks. 
null-responders to dual therapy. Preliminary efficacy results reported respectively $89 \%$ and $80 \%$ SVR 4 rates following a 12-week treatment course with or without Rbv. ${ }^{13}$

The combination of 12 or 24 weeks of SOF plus the NS3 inhibitor simeprevir was evaluated by the COSMOS Phase II study, where an overall $93 \%$ SVR12 rate in non-cirrhotic HCV-1 previous null-responder to PegIFN/Rbv was obtained. Interim analysis of a second cohort including cirrhotic HCV-1 naïve and null-responder patients showed 100\% SVR4 with the 12 -week regimen. ${ }^{17}$

Concerning only the HCV-4 patient population, a pilot study evaluated the IFN-free combination of SOF/Rbv for 12 or 24 weeks in HCV-4 treatment-naïve or -experienced patients of Egyptian ancestry, showing respectively $79 \%$ SVR12 in naïve and 59\% in experienced patients in the 12-week arms, while SVR12 for the 24-week regimens are still pending. ${ }^{18}$

\section{Resistance}

In all Phase III studies, SOF confirmed its potent antiviral activity and high resistance barrier, as $99 \%$ of patients achieved HCV-RNA undetectability by treatment week 4 and all virological failures were due to relapse. S282T has been identified as the primary mutation leading to a 4- to 24-fold decrease in susceptibility to SOF for all tested genotypes. In the ELECTRON Phase II trial, the S282T substitution was detected in a single HCV-2 subject who received SOF monotherapy for 12 weeks and relapsed 4 weeks after the end of treatment. The sample had a mean 13.5-fold reduced susceptibility to SOF; however, the mutation was no longer detectable 12 weeks after the end of treatment by next-generation sequencing analysis. ${ }^{8}$ In Phase III studies, S282T mutation was not detected either in baseline samples or at the time of relapse in any patient. Other treatment-emergent NS5B substitutions (L159F, E341D, L320F) were frequently observed in patients relapsing to SOF-based regimens; however, none were associated with a phenotypic change in SOF or Rbv susceptibility. ${ }^{9,10}$

\section{Predictors of treatment outcome with SOF-based regimens}

In the era of PegIFN plus Rbv dual therapy, many host factors have been identified as predictors affecting treatment outcome in addition to viral genotype, such as sex, age, ethnicity, obesity, insulin-resistance, coinfections (HIV), and liver fibrosis stage. ${ }^{19}$ More recently, the discovery of the single nucleotide polymorphism (SNP) rs12979860 near the interleukin 28B (IL28B) region as the strongest baseline predictor of SVR to PegIFN plus Rbv in HCV-1,4 and, to a lesser extent, in $\mathrm{HCV}-2,3$, represented a turning point in treatment outcome prediction, leading to better treatment individualization. ${ }^{20}$

This scenario is expected to gradually change when moving to DAAs, as the increased viral potency will eventually overcome the role of some host predictors, which affect treatment outcome in a relevant manner only when considering regimens with suboptimal efficacy. This was only partially true with telaprevir and boceprevir, whose antiviral efficacies were still driven by that of the PegIFN plus Rbv backbone; instead, when considering SOF-based regimens,

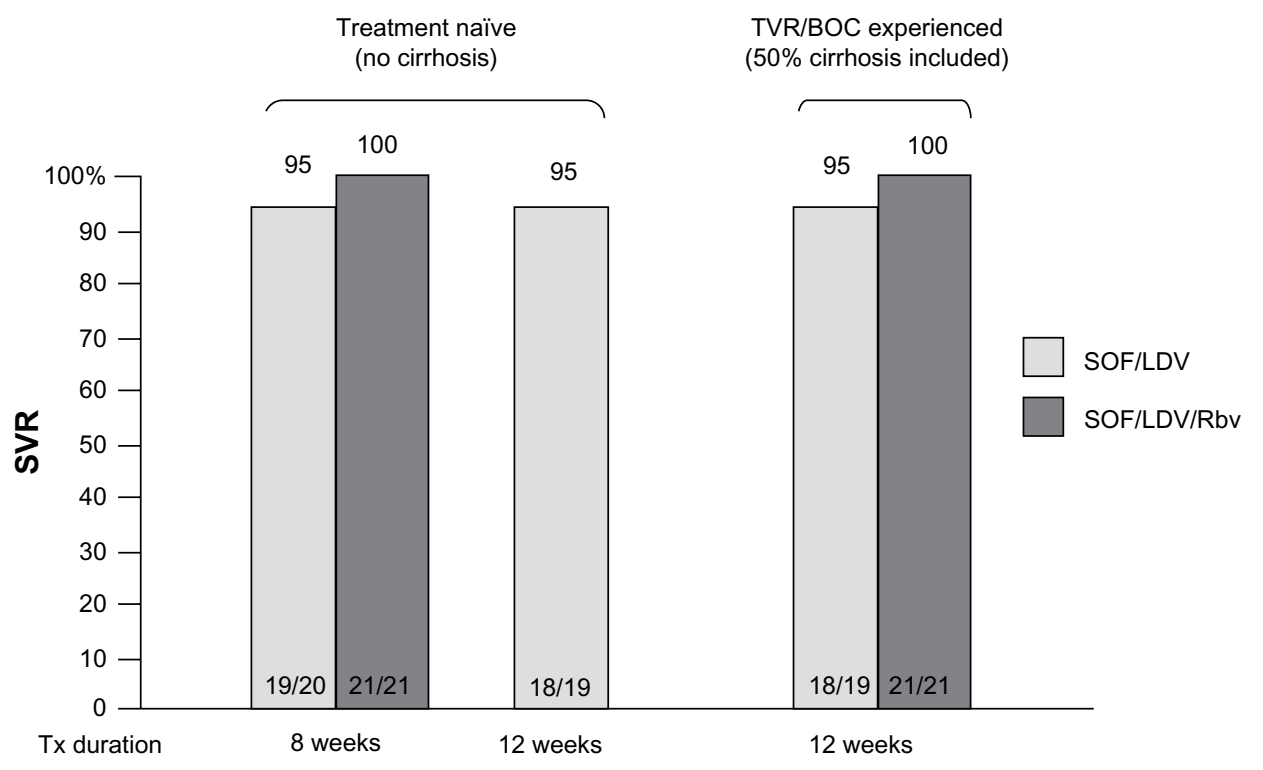

Figure 3 Sofosbuvir + ledipasvir \pm ribavirin in HCV-I patients: the LONESTAR trial.

Abbreviations: BOC, boceprevir; LDV, ledipasvir; Rbv, ribavirin; SOF, sofosbuvir; SVR, sustained virological response; TVR, telaprevir; tx, treatment. 
a retrospective analysis of registration trials demonstrated that the historically negative predictors of response to IFN-based regimens did not significantly affect SVR rates. Indeed, when analyzing patients by the presence/absence of historically negative predictors such as cirrhosis, age $\geq 65$ years, obesity, IL28B TT genotype, diabetes, black race, and high baseline viral load ( $\geq 10^{7} \mathrm{IU} / \mathrm{mL}$ ), SVR rates were independent of all predictors except for cirrhosis in $\mathrm{HCV}-3$ patients, which has been already discussed above. ${ }^{21}$

\section{Drug-to-drug interactions (DDIs)}

SOF is a substrate of drug transporters P-gp and breast cancer resistance protein (BCRP), whereas this is not true for the metabolite GS-331007. Co-administration studies showed no clinically significant interaction with cyclosporine A (CsA) or tacrolimus (TAC). High-dose CsA increased systemic SOF exposure (AUC), with $\sim 10 \%$ increase in total drug-related material with no increase in nucleotide metabolite. Based on these results, CsA or TAC can be co-administered with SOF without dose modification. ${ }^{22}$ No clinically significant DDIs were observed between SOF and the following drugs: tenofovir, efavirenz, darunavir/ritonavir, raltegravir, emtricitabine, and rilpivirine, so co-administration in HIV-infected patients is feasible. Also, methadone did not display clinically relevant DDIs with $\mathrm{SOF}^{23}$

Finally, potential DDIs between SOF and hormonal oral contraceptives have also been evaluated: co-administration with norgestimate/ethinyl estradiol was safe and welltolerated, without significant alterations in PK data. ${ }^{24}$ Based on these observations, no loss in contraceptive efficacy is expected and concomitant use with SOF is allowed.

\section{SOF in special populations: orthotopic liver transplantation (OLT) and HIV}

HCV graft reinfection occurs in all HCV-RNA positive patients at the time of liver transplantation and is associated with reduced survival due to disease recurrence, and is characterized by rapid fibrosis progression and results in poor graft outcome. Anti-HCV treatment in the pre- and post-transplant phase has always been hampered by several limitations with PegIFN-based regimens: treatment exclusion because of hepatic decompensation and risk of lifethreatening infections in the pre-transplant, while, in the post-transplant, difficult treatment management due to drug interactions with immunosuppressants and high rates of side effects that translate to reduced efficacy. In this scenario, development of IFN-free regimens with high tolerability and efficacy without DDIs would translate to extending treatment access in pre- and post-OLT patients, consequently preventing $\mathrm{HCV}$ recurrence and disease progression.

Results from a multicenter study in 61 OLT candidates for hepatocellular carcinoma (HCC) and HCV-related cirrhosis showed prevention of graft reinfection in $64 \%$ of patients treated with SOF/Rbv up to 48 weeks before liver transplantation and testing HCV-RNA below the limit of quantification (LLOQ) at the time of liver transplant. The strongest predictor of post-transplant virological response was the number of consecutive days of HCV-RNA undetectability. ${ }^{25}$ It has to be pointed out that the study was conducted in a selected patient population with Child score $\leq 7$, which is consequently not entirely representative of the HCV pre-transplant patient population. More studies are needed to evaluate the safety and efficacy of SOF-based regimens, especially in the setting of more severe hepatic decompensation and end-stage liver disease.

Concerning antiviral treatment in post-OLT, preliminary results with SOF/Rbv treatment for 24 weeks in patients with recurrent HCV hepatitis after liver transplantation have been recently presented to the AASLD Meeting: in a small patient population (40 patients included, mainly HCV-1a or $1 \mathrm{~b}, 63 \%$ with bridging fibrosis or cirrhosis), treatment was safe and well-tolerated. No significant interactions with common immunosuppressants were observed and no episodes of rejection occurred. Overall SVR4 rates were $77 \%$, while SVR12 efficacy data are still awaited. ${ }^{26}$

Preliminary data are also available on compassionate SOF use in 44 OLT patients with severe HCV recurrence: treatment with $\mathrm{SOF} / \mathrm{Rbv} \pm$ PegIFN for $\leq 48$ weeks showed significant improvement in liver function tests and clinical outcomes such as reduction in episodes of decompensation and hepatic encephalopathy in $64 \%$ of patients. After 24 weeks of treatment, $64 \%-83 \%$ of patients achieved HCVRNA undetectability and SVR12 rates ranged between $50 \%$ and $60 \%$. High mortality rates (25\%), mainly due to disease progression, were the result of treating a patient population with severe HCV recurrence, with $47 \%$ with a histological diagnosis of fibrosing cholestatic hepatitis, which is typically characterized by poor short-term outcome. ${ }^{27}$

HIV-HCV coinfected patients represent another urgent-to-treat patient population, as HIV coinfection is a known predictor of faster fibrosis progression and poor long-term outcome. The PHOTON-1 study in 182 HCV-1-3 patients receiving SOF/Rbv for $24(\mathrm{HCV}-1)$ or 12 weeks (HCV-2/3) showed overall 76\%, 88\%, and 67\% SVR12 rates, respectively. No significant changes in CD4 T cell 
Table 3 Sofosbuvir-based regimens according to HCV genotype and date of FDA approval

\begin{tabular}{ll}
\hline Regimen & $\begin{array}{c}\text { Expected FDA } \\
\text { approval date }\end{array}$ \\
\hline HCV-I & \\
IFN sparing & 2014 \\
- SOF + PegIFN/Rbv I2 weeks & \\
IFN free & 2015 (off-label) \\
- SOF + DCV I2/24 weeks & 2015 \\
- SOF + LDV 8/I2 weeks & 2014 (off-label) \\
- SOF + SMV I2/24 weeks & \\
HCV-2/3 & \\
IFN free & 2014 \\
- SOF + Rbv I2 weeks (HCV-3: I6/24 weeks) \\
- SOF + PegIFN/Rbv I2 weeks (HCV-3 only) \\
HCV-4 \\
IFN sparing \\
- SOF + PegIFN/Rbv I2 weeks \\
IFN free & \\
- SOF + Rbv I2/24 weeks & \\
\hline
\end{tabular}

Abbreviations: DCV, daclatasvir; FDA, US Food and Drug Administration; HCV, hepatitis C virus; LDV, ledipasvir; PegIFN, Peginterferon; Rbv, ribavirin; SOF, sofosbuvir; SMV, simeprevir.

count were reported and treatment was well-tolerated, with no interactions between SOF and multiple different antiretroviral regimens. ${ }^{28}$ More trials are expected to confirm these data in larger populations.

\section{Conclusion}

The development of IFN-free or sparing regimens represents a breakthrough novelty in the history of anti-HCV treatment. The next few years are expected to radically modify chronic hepatitis $\mathrm{C}$ scenarios, as access to safe and potent therapies will translate to the simplification of treatment management with the possibility to include formerly PegIFN-contraindicated patient populations. Increased treatment efficacy will also revolutionize clinicians' approach to patient counseling, as previous models for treatment individualization and outcome predictors with PegIFN plus Rbv will be mostly overcome. SOF will be one of the first DAAs in this era (Table 3) and its commercialization will be the key moment to address some open questions with these new regimens, as the huge raise in treatment costs will represent the first problem to solve for national health care services. Consequently, affordability could be the driving force for the development of new strategies in treatment individualization, where some "old" but "sufficiently effective" regimens could still represent an important option in selected patient populations.

\section{Disclosure}

Alessio Aghemo is on the advisory committee for Roche and has received travel support from BMS, GlaxoSmith Kline, and Bayer. Elisabetta Degasperi has no financial disclosures to declare.

\section{References}

1. Ghany MG, Strader DB, Thomas DL, Seeff LB. Diagnosis, management, and treatment of hepatitis C: an update. Hepatology. 2009;49: 1335-1374.

2. European Association for the Study of the Liver. EASL Clinical Practice Guidelines: management of hepatitis C virus infection. J Hepatol. 2011;55:245-264.

3. Aghemo A, Degasperi E, Colombo M. Directly acting antivirals for the treatment of chronic hepatitis $\mathrm{C}$ : unresolved topics from registration trials. Dig Liver Dis. 2013;45:1-7.

4. D'Ambrosio R, Colombo M. Safety of direct antiviral agents in real life. Dig Liver Dis. 2013;45:S363-S366.

5. Aghemo A, De Francesco R. New horizons in hepatitis $C$ antiviral therapy with direct-acting antivirals. Hepatology. 2013;58:428-438.

6. Martel-Lafferriere V, Dieterich DT. GS-7977: a promising nucleotide ana$\log$ NS5B polymerase inhibitor of HCV. Future Virol. 2012; 7: 537-546.

7. Kirby B, Gordi T, Symonds WT, Kearney BP, Mathias A. Population pharmacokinetics of sofosbuvir and its major metabolite (GS-331007) in healthy and HCV-infected adult subjects. 64th Annual Meeting of the American Association for the Study of Liver Diseases; November 1-5, 2013; Washington, DC.

8. Gane EJ, Stedman CA, Hyland RH, et al. Nucleotide polymerase inhibitor sofosbuvir plus ribavirin for hepatitis C. $N$ Engl J Med. 2013;368:34-44.

9. Lawitz E, Mangia A, Wyles D, et al. Sofosbuvir for previously untreated chronic hepatitis C infection. N Engl J Med. 2013;368:1878-1887.

10. Jacobson IM, Gordon SC, Kowdley KV, et al. Sofosbuvir for hepatitis C genotype 2 or 3 in patients without treatment options. N Engl J Med. 2013;368:1867-1877.

11. Zeuzem S, Dusheiko GM, Salupere R, et al. Sofosbuvir + ribavirin for 12 or 24 weeks for patients with HCV genotype 2 or 3: the VALENCE Trial. 64th Annual Meeting of the American Association for the Study of Liver Diseases; November 1-5, 2013; Washington, DC.

12. Lawitz E, Poordad F, Brainard DM, et al. Sofosbuvir in combination with PegIFN and ribavirin for 12 weeks provides high SVR rates in $\mathrm{HCV}$-infected genotype 2 or 3 treatment-experienced patients with and without compensated cirrhosis: results from the LONESTAR-2 Study. 64th Annual Meeting of the American Association for the Study of Liver Diseases; November 1-5, 2013; Washington, DC.

13. Gane E, Stedman CA, Hyland RH, et al. Once daily sofosbuvir/ledipasvir fixed dose combination with or without ribavirin: the ELECTRON trial. 64th Annual Meeting of the American Association for the Study of Liver Diseases; November 1-5, 2013; Washington, DC.

14. Kowdley KV, Lawitz E, Crespo I, et al. Sofosbuvir with pegylated interferon alfa-2a and ribavirin for treatment-naive patients with hepatitis C genotype-1 infection (ATOMIC): an open-label, randomised, multicentre phase 2 trial. Lancet. 2013;381:2100-2107.

15. Sulkovski MS, Gardiner DF, Rodriguez-Torres M, et al. Sustained virologic response with daclatasvir plus sofosbuvir \pm ribavirin in chronic HCV genotype 1-infected patients who previously failed telaprevir or boceprevir. 64th Annual Meeting of the American Association for the Study of Liver Diseases; November 1-5, 2013; Washington, DC.

16. Lawitz E, Poordad FF, Pang PS, et al. Sofosbuvir and ledipasvir fixeddose combination with and without ribavirin in treatment-naive and previously treated patients with genotype 1 hepatitis $\mathrm{C}$ virus infection (LONESTAR): an open-label, randomised, phase 2 trial. Lancet. 2014;383(9916):515-523.

17. Lawitz E, Ghalib R, Rodriguez-Torres M, et al. SVR results of a oncedaily regimen of simeprevir (TMC435) plus sofosbuvir (GS-7977) with or without ribavirin in cirrhotic and non-cirrhotic HCV genotype 1 treatment-naive and prior null responder patients: the COSMOS study. 64th Annual Meeting of the American Association for the Study of Liver Diseases; November 1-5, 2013; Washington, DC. 
18. Ruane PJ, Ain D, Raid J, et al. Sofosbuvir plus ribavirin in the treatment of chronic HCV genotype 4 infection in patients of Egyptian ancestry. 64th Annual Meeting of the American Association for the Study of Liver Diseases; November 1-5, 2013; Washington, DC.

19. Asselah T, Estrabaud E, Bieche I, et al. Hepatitis C: viral and host factors associated with non-response to pegylated interferon plus ribavirin. Liver Int. 2010;30:1259-1269.

20. Thompson AJ, Muir AJ, Sulkowski MS, et al. Interleukin-28B polymorphism improves viral kinetics and is the strongest pretreatment predictor of sustained virological response in genotype 1 hepatitis $\mathrm{C}$ virus. Gastroenterology. 2010;139:120-129.

21. Mangia A, Kugelmas M, Everson G, et al. Virologic response rates to Sofosbuvir-containing regimens are similar in patients with and without traditional negative predictive factors: a retrospective analysis of Phase 3 data. 64th Annual Meeting of the American Association for the Study of Liver Diseases; November 1-5, 2013; Washington, DC.

22. Mathias A, Cornpropst M, Clemons D, et al. No clinically significant pharmacokinetic drug-drug interactions between Sofosbuvir (GS-7977) and the immunosuppressants cyclosporine A or Tacrolimus in healthy volunteers. 64th Annual Meeting of the American Association for the Study of Liver Diseases; November 1-5, 2013; Washington, DC.

23. Kirby B, Mathias A, Rossi S, Moyer C, Shen G, Kearney BP. No clinically significant pharmacokinetic drug interactions between sofosbuvir (GS-7977) and HIV antiretrovirals Atripla ${ }^{\circledR}$, rilpivirine, darunavir/ritonavir, or raltegravir in healthy volunteers. 64th Annual Meeting of the American Association for the Study of Liver Diseases; November 1-5, 2013; Washington, DC.
24. German P, Moorehead L, Pang PS, Vimal M, Mathias A. Lack of a clinically important pharmacokinetic interaction between norgestimate/ ethinyl estradiol and sofosbuvir or ledipasvir in HCV-uninfected female subjects. 64th Annual Meeting of the American Association for the Study of Liver Diseases; November 1-5, 2013; Washington, DC.

25. Curry MP, Forns X, Chung R, et al. Pretransplant sofosbuvir and ribavirin to prevent recurrence of $\mathrm{HCV}$ infection after liver transplantation. 64th Annual Meeting of the American Association for the Study of Liver Diseases; November 1-5, 2013; Washington, DC.

26. Charlton M, Gane E, Manns MP, et al. Sofosbuvir and Ribavirin for the Treatment of Established Recurrent Hepatitis C Infection After Liver Transplantation: Preliminary Results of a Prospective, Multicenter Study. 64th Annual Meeting of the American Association for the Study of Liver Diseases; November 1-5, 2013; Washington, DC.

27. Forns X, Fontana RJ, Moonka D, et al. Initial evaluation of the sofosbuvir compassionate use program for patients with severe recurrent $\mathrm{HCV}$ following liver transplantation. 64th Annual Meeting of the American Association for the Study of Liver Diseases; November 1-5, 2013 Washington, DC.

28. Sulkowski M, Rodriguez-Torres M, Lalezari J, et al. All-oral therapy with sofosbuvir plus ribavirin for the treatment of HCV genotype 1, 2, and 3 infection in patients co-infected with HIV (PHOTON-1). 64th Annual Meeting of the American Association for the Study of Liver Diseases; November 1-5, 2013; Washington, DC.
Hepatic Medicine: Evidence and Research

\section{Publish your work in this journal}

Hepatic Medicine: Evidence and Research is an international, peerreviewed, open access journal covering all aspects of adult and pediatric hepatology in the clinic and laboratory including the following topics: Pathology, pathophysiology of hepatic disease; Investigation and treatment of hepatic disease; Pharmacology of drugs used for

\section{Dovepress}

the treatment of hepatic disease. Issues of patient safety and quality of care will also be considered. The manuscript management system is completely online and includes a very quick and fair peer-review system, which is all easy to use. Visit http://www.dovepress.com/ testimonials.php to read real quotes from published authors.

Submit your manuscript here: http://www.dovepress.com/hepatic-medicine-evidence-and-research-journal 